\title{
A role for the transducer of the Hippo pathway, TAZ, in the development of aggressive types of endometrial cancer
}

\author{
Laura Romero-Pérez ${ }^{1}$, Pablo Garcia-Sanz ${ }^{2,3}$, Alba Mota ${ }^{2,3}$, Susanna Leskelä ${ }^{4}$, \\ Marta Hergueta-Redondo², Juan Díaz-Martín ${ }^{1}$, M Ángeles López-García1 ${ }^{1}$, \\ M Ángeles Castilla ${ }^{1}$, Ángel Martínez-Ramírez ${ }^{5}$, Robert A Soslow ${ }^{6}$, Xavier Matias-Guiu ${ }^{7}$,
} Gema Moreno-Bueno 2,3 and Jose Palacios ${ }^{1,4}$

\begin{abstract}
${ }^{1}$ Department of Pathology, Instituto de Biomedicina de Sevilla (IBiS), Hospital Universitario Virgen del Rocío/ CSIC/Universidad de Sevilla, Sevilla, Spain; ${ }^{2}$ Department of Biochemistry, Universidad Autónoma de Madrid (UAM), Instituto de Investigaciones Biomédicas 'Alberto Sols' (CSIC-UAM), IdiPAZ, Madrid, Spain;

${ }^{3}$ Translational Research Laboratory, MD Anderson International Foundation, Madrid, Spain; ${ }^{4}$ Department of Pathology, Hospital Universitario Ramón y Cajal, Instituto de Investigación Sanitaria Ramón y Cajal (IRYCIS), Universidad de Alcalá, Madrid, Spain; ${ }^{5}$ Department of Molecular Cytogenetics, MD Anderson Cancer Center, Madrid, Spain; ${ }^{6}$ Department of Pathology, Memorial Sloan-Kettering Center, Memorial Hospital, New York, NY, USA and ${ }^{7}$ Department of Pathology and Molecular Genetics, Hospital Universitari Arnau de Vilanova, University of Lleida, IRBLleida, Lleida, Spain
\end{abstract}

\begin{abstract}
Although TAZ, the final effector of the Hippo pathway that modulates epithelial to mesenchymal transition and stemness, has been implicated in the development of different types of cancer, its role in endometrial cancer has not yet been studied. Thus, we evaluated the expression of TAZ in different types of endometrial cancer by immunohistochemistry. TAZ expression was detected in $76 \%$ of undifferentiated endometrial carcinomas, $54 \%$ of endometrial carcinosarcomas, $46 \%$ of endometrial serous carcinomas, $36 \%$ of grade 3 endometrioid carcinomas, and $18 \%$ of grade 1-2 endometrioid carcinomas, with statistically significant differences. We analyzed the WWTR1 gene that encodes TAZ by FISH and MassARRAY spectrometry, ruling out gene amplification and differential promoter methylation as the main mechanisms that modulate TAZ expression in endometrial tumors. However, we did detect a significant association between Scribble hypoexpression and delocalization with TAZ expression. Moreover, we demonstrated that TAZ promoted invasiveness, and it favored cell motility and tumor growth, in endometrial cancer cell lines. In addition, TAZ expression was associated with the transition from an epithelial to mesenchymal phenotype, both in vitro and in human tumors. Together, these data reveal a previously unknown role for TAZ and the Hippo pathway in the progression of aggressive subtypes of endometrial cancer.
\end{abstract}

Modern Pathology (2015) 28, 1492-1503; doi:10.1038/modpathol.2015.102; published online 18 September 2015

In developed countries, endometrial carcinoma is the most common malignant tumor of the female genital tract. ${ }^{1}$ On the basis of epidemiological,

Correspondence: Dr G Moreno-Bueno, PhD, Department of Biochemistry, Universidad Autónoma de Madrid (UAM), Instituto de Investigaciones Biomédicas 'Alberto Sols' (CSIC-UAM), Calle Arturo Duperier, 4, Madrid 28029, Spain or Dr J Palacios, MD, $\mathrm{PhD}$, Department of Pathology, Hospital Universitario Ramón y Cajal, Instituto de Investigación Sanitaria Ramón y Cajal (IRYCIS), Universidad de Alcalá, Carretera de Colmenar Viejo, km. 9,100, Madrid 28034, Spain.

E-mail: gmoreno@iib.uam.es or jose.palacios@salud.madrid.org Received 7 May 2015; revised 21 July 2015; accepted 23 July 2015; published online 18 September 2015 clinical, pathological, and molecular features, endometrial carcinoma can be classified into at least two main categories: ${ }^{2}$ type I estrogen-dependent carcinomas that account for $80-85 \%$ of cases and that are typically represented by low-grade (grade 1 and 2) endometrioid carcinomas, and type II endometrial carcinomas that are not estrogen dependent and that are mainly represented by a serous subtype but also by other high-grade histological tumors like clear cell or undifferentiated carcinomas. ${ }^{2}$

In endometrial carcinoma, the epithelial to mesenchymal transition has been associated with high-grade and aggressive features. ${ }^{3-6}$ Epithelial to 
mesenchymal transition is a key developmental program by which polarized epithelial cells convert into motile mesenchymal ones, and it is a process that is activated during cancer invasion and metastasis. ${ }^{7}$ The epithelial to mesenchymal transition is coordinated by a network of transcription factors that regulate the expression of proteins involved in cell polarity, cell-cell contacts, cytoskeletal structure, and extracellular matrix degradation. ${ }^{8}$ The loss of E-cadherin expression is a crucial event in epithelial to mesenchymal transition and indeed, transcription factors associated to epithelial to mesenchymal transition can be grouped according to their ability to directly or indirectly repress E-cadherin. ${ }^{9}$ Among the direct repressors, we can find zinc finger proteins like SNAIL1, SNAIL2, ZEB1 (TCF8), ZEB2 (SIP1), and the bHLH factor E47 (TCF3) or KLF8. By contrast, indirect repressors include TWIST bHLH proteins or E22. ${ }^{8}$ In addition, the epithelial to mesenchymal transition program is closely linked to the acquisition of stem cell properties in other tumor cells, as defined by the acquisition of the CD $44^{\text {high/CD }} 24^{\text {low }}$ phenotype, in vitro self-renewal and in vivo tumor initiation capacities. ${ }^{7,10}$ The epithelial to mesenchymal transition has also been implicated in the dissemination of primary tumors owing to invasion or intravasation, as well as to the generation of distant tumors by migrating cancer stem cells. ${ }^{11}$

The Hippo pathway has been implicated in epithelial to mesenchymal transition and stemness, ${ }^{12}$ and it is a pathway that coordinates cell proliferation, apoptosis, and differentiation associated with the regulation of organ development and regeneration. ${ }^{13}$ The pathway involves a conserved kinase cascade, and the final transcriptional co-activators TAZ and YAP have been implicated in tissue homeostasis and the control of organ size through tissue-specific stem cell regulation. ${ }^{14}$ Dysregulation of the Hippo pathway is correlated with epithelial to mesenchymal transition and cancer development, mainly driven by TAZ and YAP. ${ }^{15}$ These two DNA-binding proteins are normally repressed by Mst2 and Lats2 phosphorylation, ${ }^{16,17}$ or they are regulated by the expression and location of the cell polarity protein Scribble. ${ }^{18}$ TAZ/YAP and epithelial to mesenchymal transition are thought to maintain a bidirectional relationship, whereby the loss of polarity and cell contacts (key events during the epithelial to mesenchymal transition process) induces the activation of both factors, which in turn participate in the epithelial to mesenchymal transition program. ${ }^{19}$ Specifically, the transcriptional co-activator TAZ (WWTR1) has been associated with the loss of E-cadherin function in breast cancer. ${ }^{18}$ and it also induces the expression of transcription factors associated to epithelial to mesenchymal transition like ZEB1 in retinal pigment epithelial cells. ${ }^{20}$ Moreover, it has been shown that TAZ may confer cancer stem cell properties to breast cancer cells, ${ }^{18}$ and it is coupled to the loss of polarity in epithelial cells through the membrane delocalization of Scribble during epithelial to mesenchymal transition. ${ }^{18}$
Although TAZ and YAP have been associated to several types of cancer, their role in endometrial carcinoma is still unknown. To gain insight into a possible role of TAZ in endometrial carcinoma, we studied the expression of TAZ and other epithelial to mesenchymal transition regulators in a series of 143 endometrial carcinoma and endometrial carcinosarcoma samples. Enhanced TAZ protein expression was observed in high-grade endometrial tumors and this increase was associated to Scribble delocalization. To further study the role of TAZ in endometrial carcinoma, we overexpressed and silenced this protein in endometrial cancer cell lines, demonstrating that increased TAZ expression is associated with cell motility, invasiveness, and tumor growth. Finally, we found that TAZ expression is associated with molecular features of epithelial to mesenchymal transition in endometrial cell lines and tumors.

\section{Materials and methods}

\section{Tumor Samples}

In this study, we analyzed 143 formalin-fixed paraffinembedded samples from 56 grade 1-2 (low grade) and 14 grade 3 (high grade) endometrioid endometrial carcinomas, 26 endometrial serous carcinomas, 21 undifferentiated endometrial carcinomas, and 26 endometrial carcinosarcomas. ${ }^{3,4,21}$ We also analyzed 33 frozen samples of 17 low-grade endometrioid endometrial carcinomas and 16 endometrial carcinosarcomas. ${ }^{21}$ Histological typing of all the samples was performed according to the World Health Organization (WHO) classification and the tissue samples were obtained from the Departments of Pathology at the Hospital Universitario Virgen del Rocío (Sevilla, Spain), Memorial Sloan-Kettering Cancer Center (New York, USA), and Hospital Universitari Arnau de Vilanova (IRBLLEIDA, Lleida, Spain). Representative areas of these samples were selected to set up six different tissue microarrays, ${ }^{22}$ and a summary of the relevant clinicopathological features is provided in Supplementary Table 1. This study was performed following the standard Spanish ethical regulations (Ley de Investigación Orgánica Biomédica, 14 July 2007) and it was approved by the ethic committees of each institution involved.

\section{Immunohistochemistry and Fluorescence In Situ Hybridization}

Tissue microarray sections were assessed by immunohistochemistry and fluorescence in situ hybridization. Immunohistochemistry was performed using the Envision method (Dako, CA, USA) with a heat-induced antigen retrieval step, and using primary antibodies raised against TAZ and Scribble (Supplementary Table 2). In order to assay WWTR1 (3q25.1) gene amplification, we performed fluorescence in situ hybridization as described 
previously by Zordan. ${ }^{23}$ We used the Spectrum green-labeled Bacterial Artificial Chromosome (RP11-126i10, chr3:150717532-150892549) from the Children Hospital Oakland Research Institute and the Spectrum red-labelled chromosome 3 enumeration probe (Vysis CEP3. Abbott).

\section{DNA Isolation and Sequenom MassARRAY Quantitative DNA Methylation Analysis}

DNA was isolated from 33 frozen tissues (17 low-grade endometrioid endometrial carcinomas and 16 endometrial carcinosarcomas). Bisulfite conversion details are shown in Supplementary Methods 1. The purified and treated DNA was amplified in a TProfessional Standard 384 Thermocycler (Biometra, Jena, Germany) using specific PCR primers (Supplementary Table 3). MassARRAY spectrometry of the PCR products was performed according to the manufacturer's protocol and the data were processed with the EpiTyper Analyser software v1.0 (Sequenom, San Diego, CA, USA).

\section{RNA Isolation, mRNA and microRNA Expression Analysis}

RNA isolation, total RNA quantification, and reverse transcription of 38 formalin-fixed paraffin-embedded endometrial carcinoma samples (11 low/high-grade endometrioid endometrial carcinomas, 10 undifferentiated endometrial carcinomas, 9 endometrial serous carcinomas, and 8 endometrial carcinosarcomas) and cell lines was performed as described in the Supplementary Methods 2. We used different TaqMan Gene Expression Assays (Applied Biosystems: Supplementary Table 4) for qRT-PCR using the SsoFastProbe Supermix (Bio-Rad) in a CFX96 Real Time PCR Detection System (Bio-Rad). The resulting data were analyzed using the $2^{-(\Delta \Delta \mathrm{Ct})}$ method to estimate gene expression. ${ }^{24}$ In addition, the expression of the miR-200 family microRNAs (miR-200a/b/c, miR-141, and miR-429: Supplementary Table 5) was analyzed by qRT-PCR with the TaqMan Universal PCR Master Mix (Applied Biosystems) on a 7900HT Fast Real Time PCR System with the SDS 2.3 software (Applied Biosystems).

\section{Cell Culture and Infection}

The function of the WWTR1 gene was studied in different endometrial cancer cell lines: HEC1A and Ishikawa, derived from a well differentiated endometrioid endometrial carcinoma; AN3CA, derived from a poor differentiated endometrial carcinoma; and SK-UT-1 and SK-UT-1B, derived from an endometrial leiomyosarcoma. To generate stable clones, TAZ overexpression in Ishikawa cells was induced by retroviral infection, and TAZ was silenced by lentiviral infection of AN3CA cells (Supplementary Methods 3).

\section{Western Blotting and Immunofluorescence}

Western blots and immunofluorescence were performed as described previously. ${ }^{25}$ The antibody details are provided in Supplementary Table $6 .^{26}$ Immunofluorescence preparations were examined using an Eclipse 90i microscope system (Nikon) and processed with Image-J analysis software.

\section{Migration and Invasion Assays}

The migration and invasion assays were performed as described previously. ${ }^{25}$ Briefly, cells were grown to 85\% confluence in 60-mm cell culture dishes, and a wound was then made in the culture by scratching the monolayer. After the times indicated, pictures of three selected fields from each plate were taken. Invasion assays were carried out on modified Boyden chambers (8- $\mu \mathrm{m}$ pore filters) coated with $250 \mu \mathrm{g} / \mathrm{ml}$ matrigel, seeding $1 \times 10^{5}$ cells and quantifying the cells on the lower part of the filter $24 \mathrm{~h}$ after seeding.

\section{In Vitro Cell Proliferation}

Cell proliferation was assayed on $1.5 \times 10^{4}$ cells grown in a 96-well plate according to the Alamar Blue protocol (resazurin: Life Technologies). A Biotek Epoch microplate spectrophotometer was used to determine absorbance at 570 and $600 \mathrm{~nm}$.

\section{In Vivo Tumorigenesis Assay}

Ishikawa or AN3CA cells $\left(1 \times 10^{6}\right)$ were injected subcutaneously into both dorsal flanks of 8-week-old female Balb/c immunocompromised mice ( $n=6$ for each condition; Charles River Laboratories). After cell injection, tumor growth was monitored weekly by measuring the two orthogonal external diameters with a calliper. When the tumors reached a size of $0.90-1 \mathrm{~cm}^{3}$, they were surgically excised and processed for histological analysis. A minimum of four tumors were analyzed for each cell line.

\section{Statistics}

Normality tests were used to assess the sample distribution and cross tables were used with a Pearson's chi-squared test to evaluate immunohistochemical differences. Pearson's correlations were evaluated to determine the association between mRNA and miRNA expression, while a Student's $t$ test, Fisher's Least Significant Difference test, or oneway ANOVA was used to compare the means of expression between groups. All analyses were performed with SPSS 17.0 for Windows (SPSS) and significance was considered at $P \leq 0.05$. 


\section{Results}

TAZ Expression Is Characteristic of High-grade Endometrial Carcinomas, Especially Undifferentiated Endometrial Carcinomas

In order to study the potential role of TAZ in endometrial carcinoma, we first analyzed its expression in different subtypes of endometrial carcinoma by immunohistochemistry. We found that TAZ expression was associated more specifically to high-grade endometrial tumors, being detected in $76 \%(16 / 21)$ of undifferentiated endometrial carcinomas, $54 \%(14 / 26)$ of endometrial carcinosarcomas, $46 \%(12 / 26)$ of endometrial serous carcinomas, and $36 \%(5 / 14)$ of high-grade endometrioid endometrial carcinomas. However, only 18\% (10/56) of low-grade endometrioid endometrial carcinomas expressed TAZ (Figure 1a and Table 1), and the difference between endometrial serous carcinoma, endometrial carcinosarcoma, or undifferentiated endometrial carcinoma and low-grade endometrioid endometrial carcinomas were significant in pairwise comparisons $(P<0.05)$. Our findings indicate that TAZ is mainly expressed in the aggressive subtypes of endometrial carcinomas.

\section{TAZ Expression Is Associated to Anomalous Scribble Expression but Not to WWTR1 Gene Amplification or Promoter Methylation}

Several mechanisms can control TAZ expression, including WWTR1 gene amplification, promoter

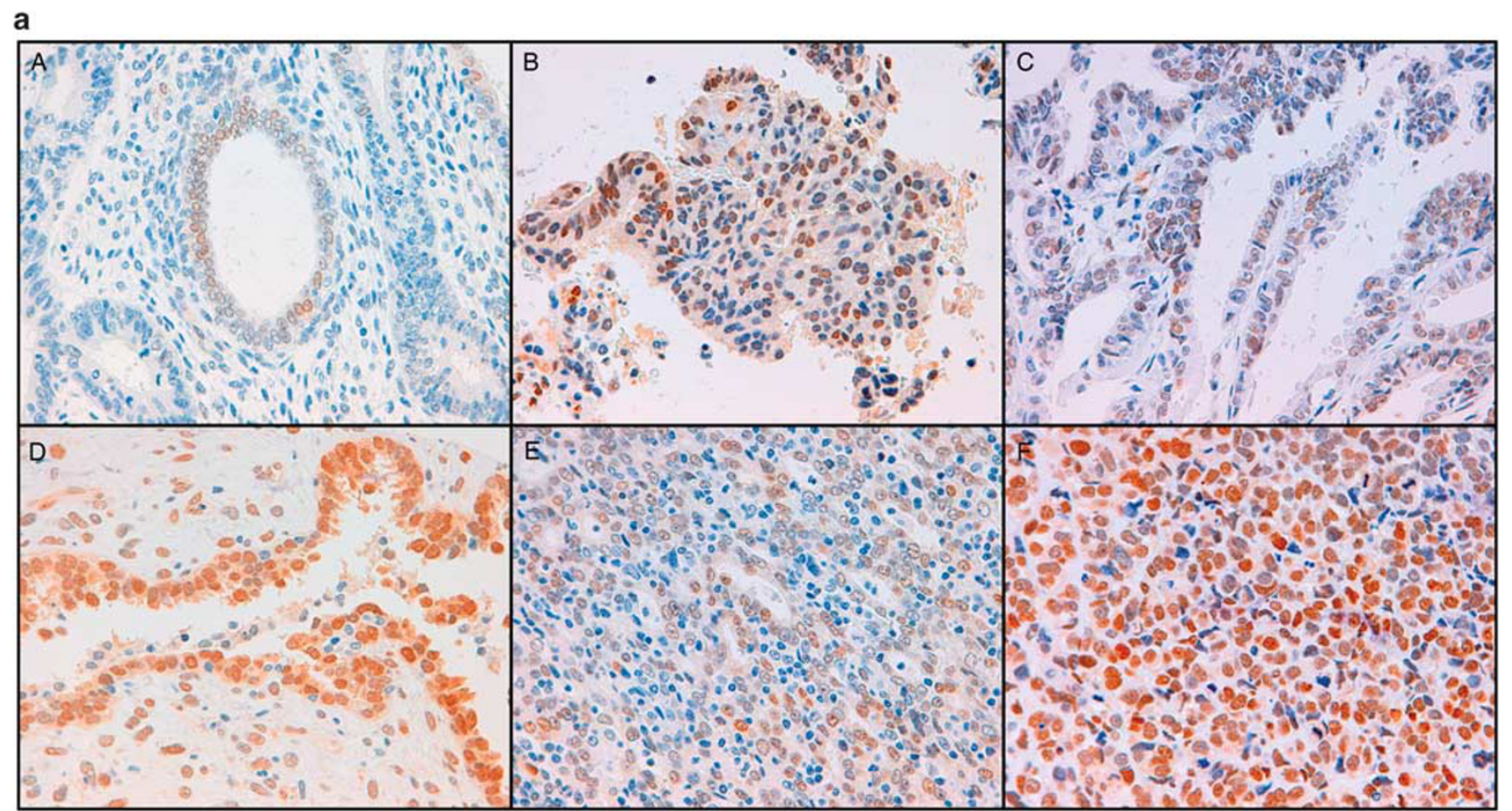

b

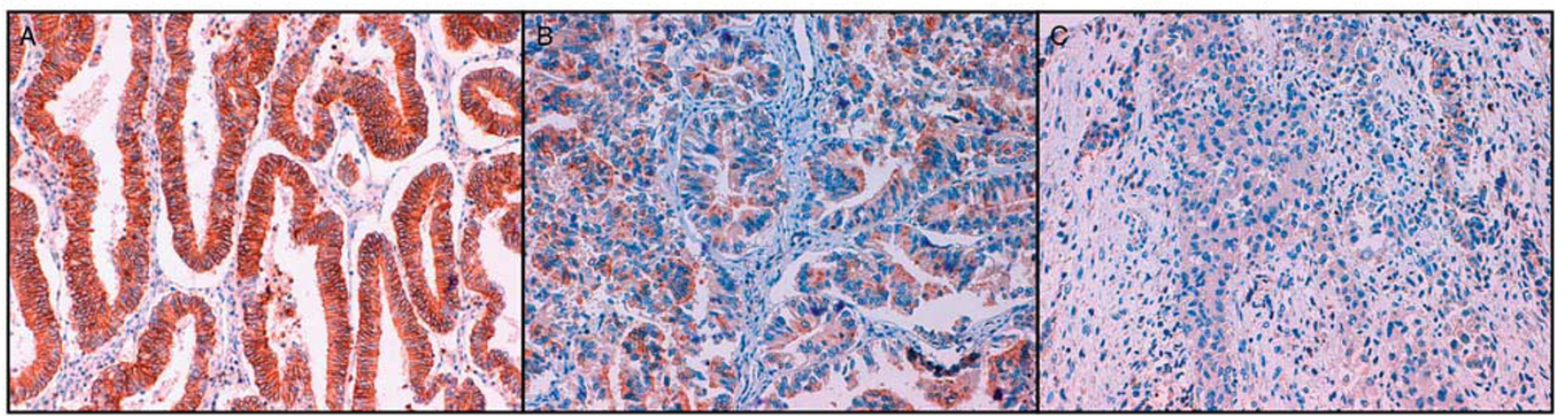

Figure 1 (a) Immunohistochemistry of the TAZ protein in different subtypes of endometrial cancer ( $\times 20)$ : (A) Low-grade endometrioid endometrial carcinoma; (B) high-grade endometrioid endometrial carcinoma; (C) endometrial serous carcinoma; (D) endometrial carcinosarcoma; (E) and (F) different intensities from positive samples of undifferentiated endometrial carcinoma. (b) Immunohistochemistry of the Scribble protein in different subtypes of endometrial cancer $(\times 20)$. (A) Membrane conserved Scribble expression in a low-grade endometrioid endometrial carcinoma. (B) Redistribution of Scribble in a low-grade endometrioid endometrial carcinoma. (C) Weak Scribble expression and redistribution in an undifferentiated endometrial carcinoma. 
Table 1 Frequency of TAZ immunoreactivity in different subtypes of endometrial cancer $(N=143)$ : low and high-grade endometrioid endometrial carcinomas (LG-EEC and HG-EEC), endometrial serous carcinoma (ESC), endometrial carcinosarcoma (ECS), and undifferentiated endometrial carcinoma (UEC)

\begin{tabular}{lc}
\hline Tumor subtype & TAZ expression \\
\hline LG-EEC & $18 \%(10 / 56)$ \\
HG-EEC & $36 \%(5 / 14)$ \\
ESC & $46 \%(12 / 26)$ \\
ECS & $54 \%(14 / 26)$ \\
UEC & $76 \%(16 / 21)$
\end{tabular}

Samples were considered as positive when TAZ expression was found in more than $5 \%$ of the tumor cells. Pairwise comparisons show differences between the low-grade endometrioid endometrial carcinomas and endometrial serous carcinoma, endometrial carcinosarcoma, or undifferentiated endometrial carcinoma group $(P<0.05)$ but not between the high-grade groups (high-grade endometrioid endometrial carcinoma, endometrial serous carcinoma, endometrial carcinosarcoma, undifferentiated endometrial carcinoma).

hypermethylation, or inactivation of the Hippo pathway. ${ }^{18,27}$ We first analyzed the WWTR1 locus (3q25.1) in tissue microarray by fluorescence in situ hybridization in order to assess whether the WWTR1 gene was amplified in endometrial carcinoma. ${ }^{18}$ We were unable to find WWTR1 amplification in any of the tumors examined and thus, we ruled out this as a possible influence on TAZ expression (Supplementary Figure 1).

Methylation could inhibit the WWTR1 promoter, and in this way, it might be associated with TAZ expression levels in endometrial carcinomas. We analyzed a $1.1 \mathrm{~kb}$ region of the WWTR 1 gene promoter in 16 endometrial carcinosarcomas and 17 low-grade endometrioid endometrial carcinomas by MassARRAY spectrometry and we were unable to find significant differences in the methylation of this promoter region in the analyzed groups. In addition, methylation status did not appear to be correlated with TAZ expression in these samples (data not shown). Similarly, we failed to detect differences in methylation among the different histotypes when the YAP1 promoter was analyzed (Supplementary Figure 2).

The expression and subcellular location of Scribble is also an important means to modulate the Hippo pathway ${ }^{18}$ and thus, we studied Scribble expression and subcellular distribution by immunohistochemistry to evaluate whether this might influence TAZ expression in endometrial carcinomas. We evaluated 28 low-grade endometrioid endometrial carcinomas, 14 high-grade endometrioid endometrial carcinomas, 26 endometrial serous carcinomas, 16 undifferentiated endometrial carcinomas, and 22 endometrial carcinosarcomas (Figure 1b). Membrane expression of Scribble was conserved in $71 \%(20 / 28)$ of low-grade endometrioid endometrial carcinomas but only in $21 \%(3 / 14)$ of high-grade endometrioid endometrial carcinomas, $27 \%(7 / 26)$ of endometrial serous carcinomas, $19 \%$
Table 2 Scribble expression and subcellular location in different subtypes of endometrial tumors $(N=106)$ : low and high-grade endometrioid endometrial carcinoma (LG-EEC and HG-EEC), endometrial serous carcinoma (ESC), endometrial carcinosarcoma (ECS), and undifferentiated endometrial carcinoma (UEC)

\begin{tabular}{lrc}
\hline $\begin{array}{l}\text { Tumor } \\
\text { subtype }\end{array}$ & $\begin{array}{c}\text { Conserved membrane } \\
\text { Scribble expression }\end{array}$ & $\begin{array}{c}\text { Reduced/delocalized } \\
\text { Scribble expression }\end{array}$ \\
\hline LG-EEC & $20 / 28(71 \%)$ & $8 / 28(29 \%)$ \\
HG-EEC & $3 / 14(21 \%)$ & $11 / 14(79 \%)$ \\
ESC & $7 / 26(27 \%)$ & $19 / 26(73 \%)$ \\
ECS & $3 / 22(14 \%)$ & $19 / 22(86 \%)$ \\
UEC & $3 / 16(19 \%)$ & $13 / 16(81 \%)$ \\
\hline
\end{tabular}

Pairwise comparisons show differences between low-grade endometrioid endometrial carcinoma and the rest of groups $(P<0.001)$ but not between the high-grade groups (high-grade endometrioid endometrial carcinoma, endometrial serous carcinoma, endometrial carcinosarcoma, undifferentiated endometrial carcinoma).

(3/16) of undifferentiated endometrial carcinomas, and $14 \%(3 / 22)$ of endometrial carcinosarcomas (Table 2). We observed significant differences between low-grade endometrioid endometrial carcinomas and the rest of the endometrial cancer subgroups $(P<0.001)$, but no differences were found when we compared high-grade endometrial tumors (high-grade endometrioid endometrial carcinomas, endometrial serous carcinomas, undifferentiated endometrial carcinomas, and endometrial carcinosarcomas). Remarkably, nuclear TAZ was frequently detected in endometrial carcinomas with weak and delocalized Scribble expression, observing a significant correlation between TAZ and Scribble expression $(P<0.05)$.

According to these data, TAZ expression is not related to WWTR1 amplification or methylation status but rather, it appears to be regulated by the expression and subcellular distribution of Scribble in endometrial carcinomas.

\section{TAZ Overexpression in Endometrial Cancer Cells Increases Early Cell Motility, Invasiveness, and Tumor Growth}

To further study the role of TAZ in endometrial tumorigenesis, we analyzed the TAZ protein and mRNA levels in a panel of endometrial cancer cell lines (Supplementary Figure 3A and 3B, respectively). Ishikawa cells are derived from a welldifferentiated endometrioid endometrial carcinoma and they express TAZ mRNA and protein relatively weakly, in agreement with our findings in human low-grade endometrioid endometrial carcinomas. By contrast, TAZ was expressed strongly in AN3CA cells, which stem from a poorly differentiated endometrial carcinoma, consistent with our observations in undifferentiated endometrial carcinomas. Hence, we selected Ishikawa and AN3CA cells to generate 
a

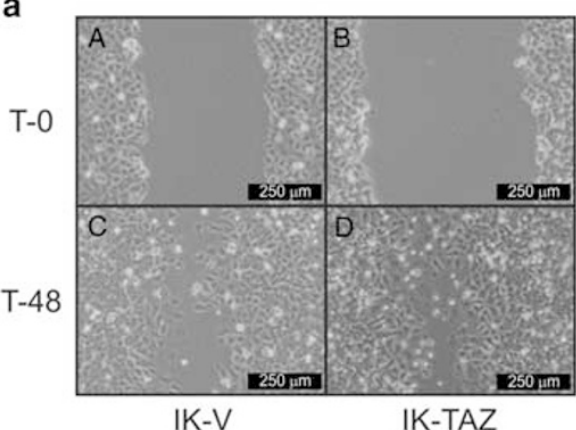

b

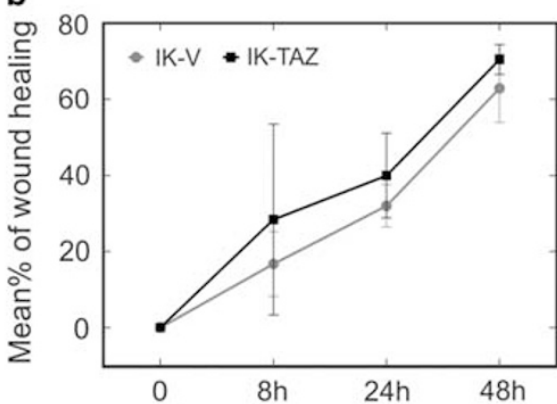

c

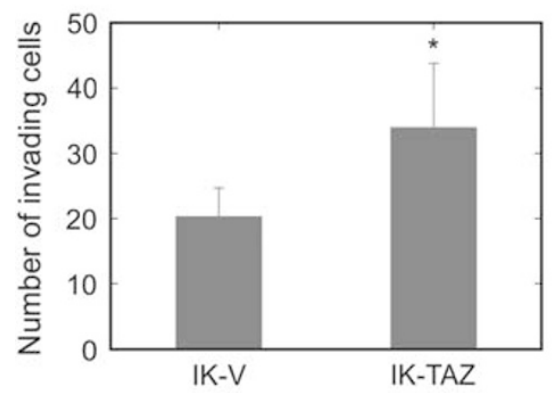

d

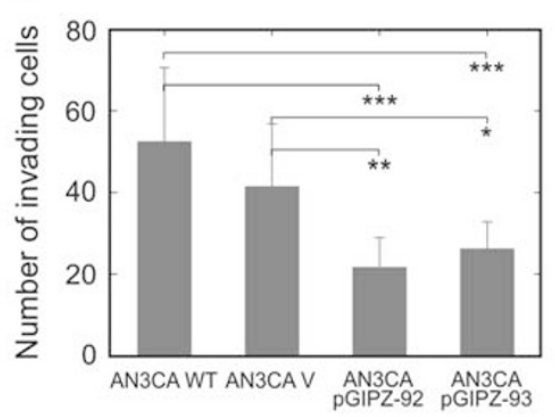

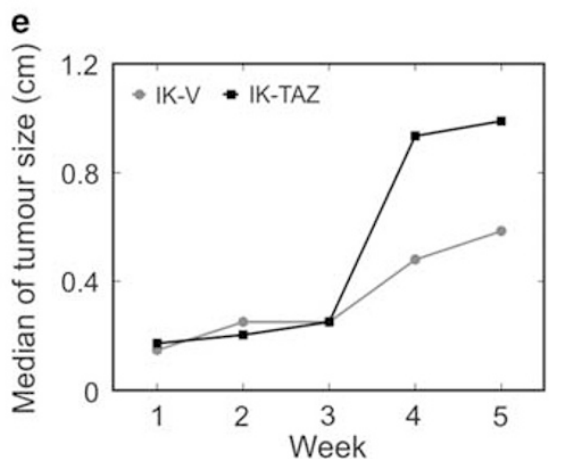

Figure 2 Changes in cell behavior when TAZ was overexpressed. (a) Images from a wound-healing assay at T-0 and T-48 h. (b) Average wound closing per clone. (c) Mean number of invasive Ishikawa-TAZ (IK-TAZ) and Ishikawa-control cells (IK-V). (d) Invasive ability of controls AN3CA cells and AN3CA-shTAZ clones: ${ }^{*} P<0.05$; ${ }^{*} P<0.01$; ${ }^{*} *{ }^{*} P<0.001$. (e) Median of tumor size (cm) over time in the subcutaneous cell injection assay.

stable TAZ-overexpressing and -silenced clones, respectively.

A wound-healing assay was used to characterize the in vitro migration and motility of the transfected cells. An increase in the migration of Ishikawa-TAZ cells was observed over $8 \mathrm{~h}$, while Ishikawa-TAZ cells closed the wound area by an average of $28.4 \%$ in this time, and the control cells closed just $16.7 \%$ of the wound. However, after $48 \mathrm{~h}$, the differences between these two cell types were smaller and both clones had completely closed the wound after nearly $72 \mathrm{~h}$ (Figure 2a and b). Although the differences were not statistically significant, these data indicated that TAZ may enhance the migratory capacity of Ishikawa cells. We did not find any significant differences in the migration of AN3CA clones in which TAZ was silenced with respect to their controls (data not shown).

When we studied the invasion capacity of the different clones, TAZ overexpression on Ishikawa cells appeared to be correlated with an increase in invasiveness $(P<0.05$, Figure 2c). By contrast, in the pGIPZ-92 and pGIPZ-93 AN3CA silenced clones, we detected fewer invading cells than in the wild-type control cells and those that received the empty pGIPZ $(P<0.05$ : Figure 2d). These data suggested that TAZ expression enhances the invasive capacity of endometrial cancer cells. Importantly, these differences in cell migration and cell invasion were not related to any alteration in cell proliferation (Supplementary Figure 4A and 4B).

To obtain further information on the biological function of TAZ in endometrial carcinoma, we studied tumorigenesis in vivo. The Ishikawa and AN3CA control cells gave rise to tumors at most injections sites $(67 \% ; 12 / 18)$ and while IshikawaTAZ cells showed a similar tumor growth rate as control cells at the outset, this had increased significantly 3 weeks after injection (Figure 2e). No histological changes were evident in the isolated lesions derived from Ishikawa-TAZ and control cells, the size of the tumor represented the only significant difference. These results suggest a new role for TAZ as a tumor growth enhancer once a primary tumor has been established. By contrast, the size of the tumors produced by AN3CA-shTAZ clones did not show differences with respect to that of the controls (data not shown).

\section{TAZ Expression Is Associated with Epithelial to Mesenchymal Transition Features in Endometrial Cancer Cell Lines and Human Tumors}

As the acquisition of a motile and tumorigenic phenotype with low proliferative activity is a feature of cells showing epithelial to mesenchymal transition, and TAZ expression promotes epithelial to mesenchymal transition in other tumors, ${ }^{16,18,27-30}$ 

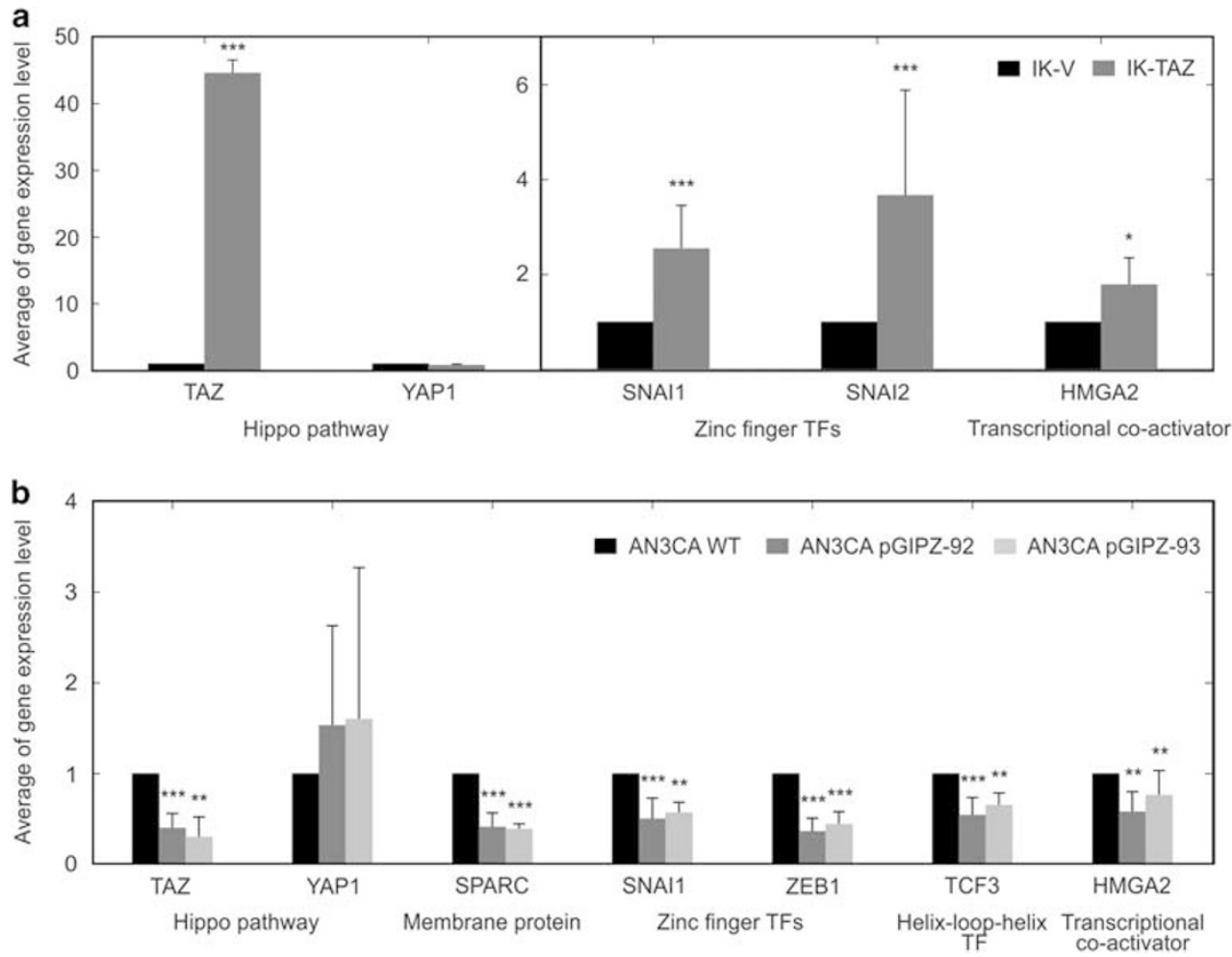

Figure 3 Changes in the expression of epithelial to mesenchymal transition-related genes upon TAZ overexpression and silencing. (a) Changes in gene expression upon TAZ overexpression in Ishikawa cells. T-test with dCT values: ${ }^{*} P<0.05 ;{ }^{* *} P<0.01$; ${ }^{* * *} P<0.001$. (b) Changes in gene expression upon TAZ silencing in AN3CA cells with two different shRNAs (pGIPZ-92 and pGIPZ-93). Least Significant Difference-test with dCT values: ${ }^{*} P<0.05 ;{ }^{*} P<0.01 ;{ }^{*} *{ }^{*} P<0.001$.

we assessed whether TAZ expression was associated with modifications of epithelial to mesenchymal transition features in endometrial cancer cells. The expression of a set of previously described epithelial to mesenchymal transition-related genes ${ }^{8}$ was evaluated in both types of endometrial cancer clones and we found that some epithelial to mesenchymal transition genes were expressed more strongly in Ishikawa-TAZ cells, such as SNAI1 $(P<0.001)$, SNAI2 $(P<0.001)$, and HMGA2 $(P<0.05$ : Figure $3 a)$. By contrast, when TAZ expression was silenced in AN3CA cells by either of the shRNAs, we observed a decrease in the expression of the membrane factor $\operatorname{SPARC}(P<0.001)$, SNAI1 $(P<0.01), Z E B 1(P<0.001)$, TCF3 $(P<0.01)$, and HMGA2 $(P<0.01$ : Figure $3 \mathrm{~b})$. These changes may reflect a role for TAZ in the induction of the epithelial to mesenchymal transition program ${ }^{19}$ in endometrial cancer cell lines.

We next analyzed the expression of several proteins linked to the epithelial to mesenchymal transition process in the clones generated. Ishikawa-TAZ cells contained less E-cadherin and more N-cadherin than their control cells (Figure 4a), consistent with the acquisition of mesenchymal features. Conversely, TAZ silencing in AN3CA cells reduced their vimentin expression and increased claudin-1 expression (Figure 4b), indicating a shift at the molecular level to a more epithelial phenotype, although no such morphological changes were observed. Because not only the expression level but also the location of some proteins may change upon the onset of epithelial to mesenchymal transition, we analyzed the subcellular distribution of TAZ and Scribble in Ishikawa-TAZ and control cells by immunofluorescence, as well as the distribution of the epithelial to mesenchymal transition-related proteins vimentin and $\beta$-catenin. TAZ overexpression not only enhanced vimentin expression but it also decreased the expression of Scribble and $\beta$-catenin, driving their translocation from the membrane to the cytoplasm (Figure 4c).

To assess the relationship between TAZ expression and epithelial to mesenchymal transition features in human endometrial carcinomas, we compared the expression of epithelial to mesenchymal transition genes between low-grade endometrioid endometrial carcinoma (representing the prototype of endometrial carcinoma with a more epithelial phenotype and the subtype in which TAZ expression is least often detected) and endometrial carcinosarcoma (an endometrial carcinoma in which a complete epithelial to mesenchymal transition has been undergone $^{3}$ and one of the subtypes that most frequently expresses TAZ). Some genes related to the epithelial to mesenchymal transition were expressed distinctly in these two types of tumor, such as CDH1, SNAI1, SNAI2, HMGA2, ZEB1, and ZEB2 $(P<0.05$ : Figure 5). Moreover, when we compared the expression of the WWTR1 gene and 
a

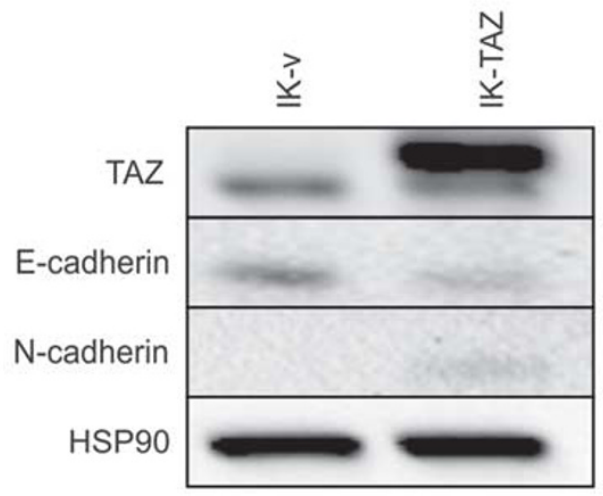

b

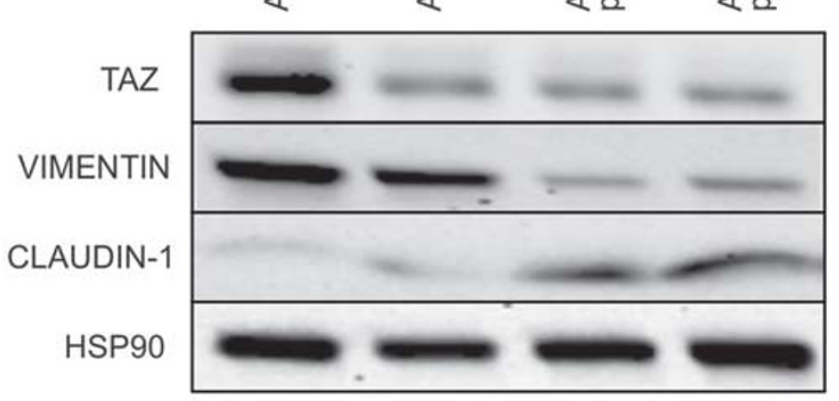

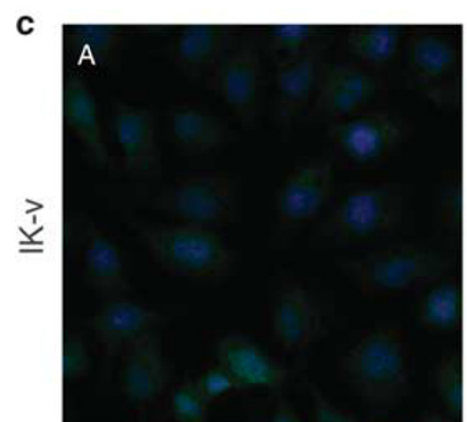

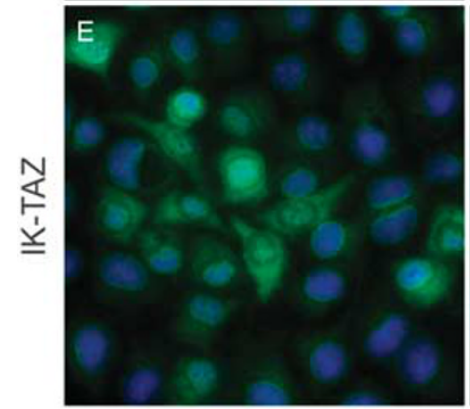

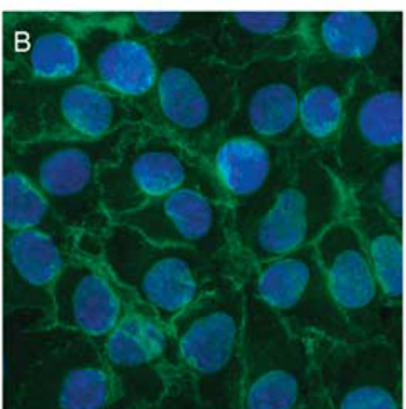

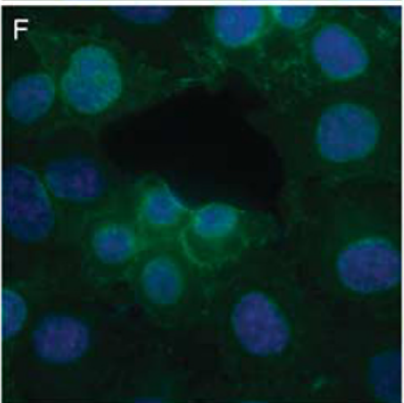

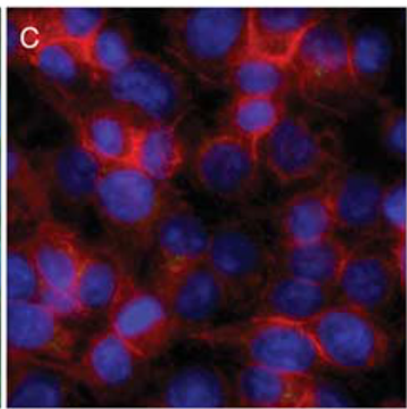

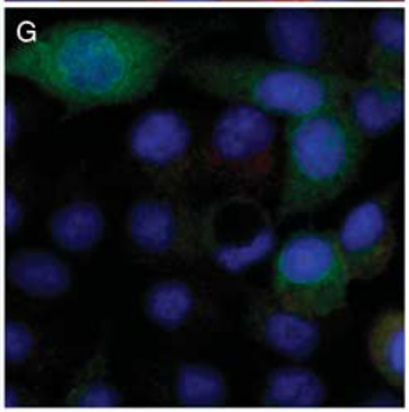

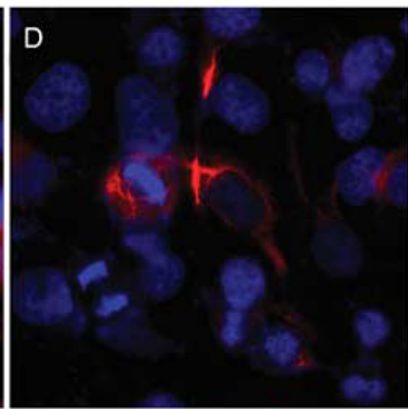

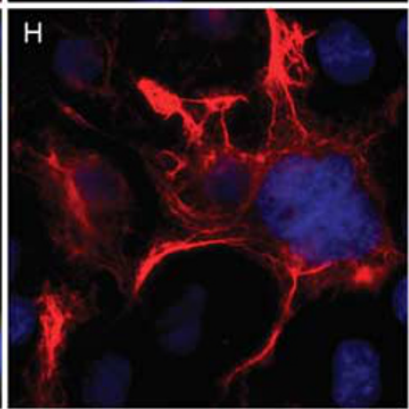

Figure 4 Changes in protein expression and localization. (a) Protein expression in Ishikawa clones assessed in western blots. (b) Protein expression in AN3CA wild type and silenced clones assessed in western blots. (c) Immunofluorescence of Ishikawa clones for different proteins: (A) and (E) TAZ (green); (B) and (F) Scribble (green); (C) and (G) B-catenin (red) and TAZ (green); (D) and (H) Vimentin (red).

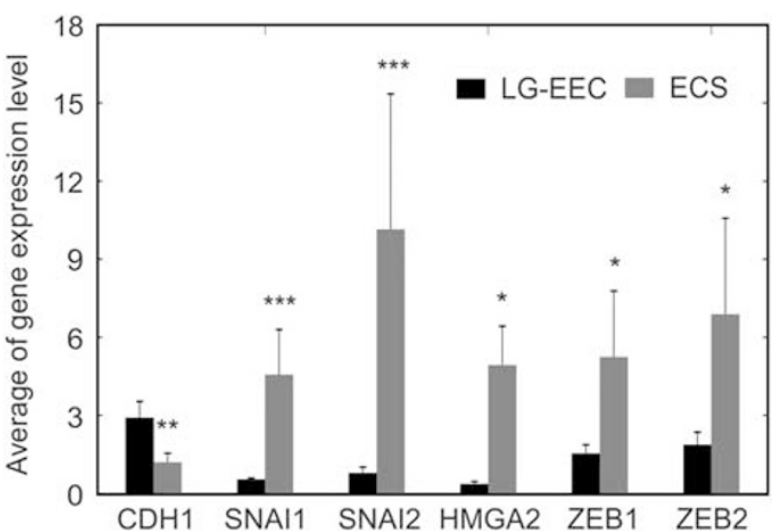

Figure 5 Epithelial to mesenchymal transition gene expression. mRNA analysis of 15 low-grade endometrioid endometrial carcinoma (LG-EEC) and 20 endometrial carcinosarcomas (ECS). T-test with dCT values: ${ }^{*} P<0.05 ;{ }^{* *} P<0.01 ; * * * P<0.001$. that of the epithelial to mesenchymal transitionrelated genes, we observed a negative correlation between WWTR1 and CDH1 $(P<0.05)$, and a positive relationship between $W W T R 1$ and SNAI1 $(P<0.01)$ or HMGA2 $(P<0.05$ : Supplementary Figure 5$)$. This pattern was consistent with the changes observed in Ishikawa-TAZ cells, and these results show that TAZ expression was associated with the expression of epithelial to mesenchymal transition-related factors and with the acquisition of epithelial to mesenchymal transition features in endometrial tumors.

We further studied how TAZ contributes to the regulation of epithelial to mesenchymal transition by analyzing the miR-200 family of microRNAs, the deregulation of which is involved in epithelial to mesenchymal transition. ${ }^{31,32}$ We found that all miR-200 microRNAs were significantly downregulated in endometrial carcinosarcomas when compared 
with low-grade endometrioid endometrial carcinomas $(P<0.01$ : Supplementary Figure 6), consistent with the mesenchymal phenotype of these tumors. When we compared microRNA expression with that of epithelial to mesenchymal transition-related genes, we found a positive relationship between the expression of all the members of miR-200 family and $C D H 1(P<0.001)$, and a negative relationship with HMGA2 $(P<0.05)$, SNAI1 $(P<0.05)$, SNAI2 $(P<0.05$, except for miR-200c $)$, TWIST1 $(P<0.05$, except for miR-200a), ZEB1 $(P<0.05$ except for miR-200b/c), and ZEB2 $(P<0.05$ for miR-200a and -141: data not shown). Interestingly, an analysis of the available gene array data from NCI60 cell lines suggested that members of the miR-200 family may target the WWTR 1 3'-UTR region. ${ }^{33}$ Accordingly, we found that WWTR1 expression was negatively correlated with that of all miR-200 microRNAs (Supplementary Figure 7): miR-141 $(P<0.001)$, miR-200a $(P<0.01)$, miR-200b $(P<0.05)$, miR-200c $(P<0.01)$, and miR-429 $(P<0.05)$.

\section{Discussion}

TAZ is a transcriptional co-activator of the Hippo pathway that is involved in epithelial to mesenchymal transition and stemness in different tumors. For instance, it has been demonstrated that TAZ is implicated in promoting migration and invasion in breast cancer cell models, ${ }^{15}$ and it has also been shown that TAZ knockdown provokes defects in the maintenance of pluripotency in embryonic stem cells. ${ }^{34}$ Hence, it would appear that TAZ is a potent activator of cell transformation and tumorigenesis. ${ }^{35,36}$ On the basis of this evidence, and given the importance of epithelial to mesenchymal transition and stemness in the carcinogenesis of certain subtypes of endometrial tumors, ${ }^{3,4}$ we analyzed TAZ expression in a series of 143 endometrial carcinoma samples. We found that TAZ expression was associated with high-grade endometrial tumors, mainly represented by undifferentiated endometrial carcinomas and endometrial carcinosarcomas.

Different mechanisms have been proposed to modulate TAZ expression in human cancers. Amplification of the WWTR1 locus (3q25.1) was detected in approximately $8 \%$ of breast cancers, ${ }^{37}$ and this was related with increased TAZ/YAP activity. ${ }^{18}$ Provisional data from The Cancer Genome Atlas (http://www.cbioportal.org) reported a 5\% amplification of WWTR1 in endometrial carcinosarcomas, however, we did not find any amplification of WWTR1 in the undifferentiated endometrial carcinoma or endometrial carcinosarcoma tissue in our series. This could reflect the low amplification frequency and the relative low number of cases analyzed.

TAZ has been closely associated with a mesenchymal signature in glioblastomas, ${ }^{27}$ and weak TAZ expression was correlated with $\mathrm{CpG}$ island hypermethylation in the WWTR1 promoter of low-grade gliomas. ${ }^{27}$ However, we did not find significant differences in the methylation status of WWTR1 and its paralogue YAP1 in endometrial carcinosarcoma and endometrioid endometrial carcinoma samples, suggesting that this modification is unlikely to control the expression of TAZ. Together, these data rule out the possibility that TAZ expression in endometrial carcinoma is regulated by gene amplification or promoter methylation as the most common or unique regulatory mechanisms.

Activation of the Hippo pathway has been proposed as one of the main mechanisms that modulates TAZ stability and its subcellular location. ${ }^{18,38}$ This pathway may be affected by mechanical stimuli such as cell contact or cytoskeletal dynamics. ${ }^{39}$ Indeed, when the cell polarity protein Scribble is located at the membrane, Hippo signalling is activated and TAZ expression repressed. However, if cell polarity is lost during tumor progression or as a result of the induction of epithelial to mesenchymal transition, Scribble becomes delocalized and consequently, enhancing TAZ expression. ${ }^{18}$ Scribble may regulate TAZ at the posttranscriptional level, because silencing Scribble in breast cancer cells increases the TAZ protein in cells without affecting its mRNA levels or their epithelial morphology. ${ }^{18}$ Our in vivo results confirm this cross-talk in endometrial carcinoma, showing that Scribble delocalization is associated with TAZ nuclear expression in high-grade endometrial tumors. Reduced and delocalized Scribble expression in endometrial carcinoma has been associated with poor prognostic factors, such as advanced stages, histopathological differentiation, and lymph node metastasis, ${ }^{40}$ in accordance with our data. Moreover, we also observed a reciprocal regulation in vitro because, in Ishikawa-TAZ cells, Scribble was translocated from the membrane to the cytoplasm. This redistribution might reflect an alteration in cell polarity as a consequence of the acquisition of mesenchymal properties upon TAZ overexpression.

We show that TAZ overexpression enhances the ability of cells to migrate, as well as produce a significant increase in their invasive capacity without affecting proliferation, suggesting that TAZ fulfills a role in the development of the epithelial to mesenchymal transition in vitro. Our in vivo tumorigenesis assay in mice also suggests that TAZ induces tumor growth once the tumor has been established. Indeed, the gene expression analysis suggests that TAZ overexpression promotes the expression of the epithelial to mesenchymal transition inducers SNAI1, SNAI2, and HMGA2, whereas the silencing of TAZ decreases that of SPARC, SNAI1, ZEB1, TCF3, and HMGA2. Accordingly, TAZ would appear to directly or indirectly regulate the transcription of these epithelial to mesenchymal transition and epithelial dedifferentiation markers. These in vitro results were confirmed in vivo, because WWTR1 expression was correlated with that of CDH1, SNAI1, and HMGA2 in the endometrial tumors analyzed. We also show that 

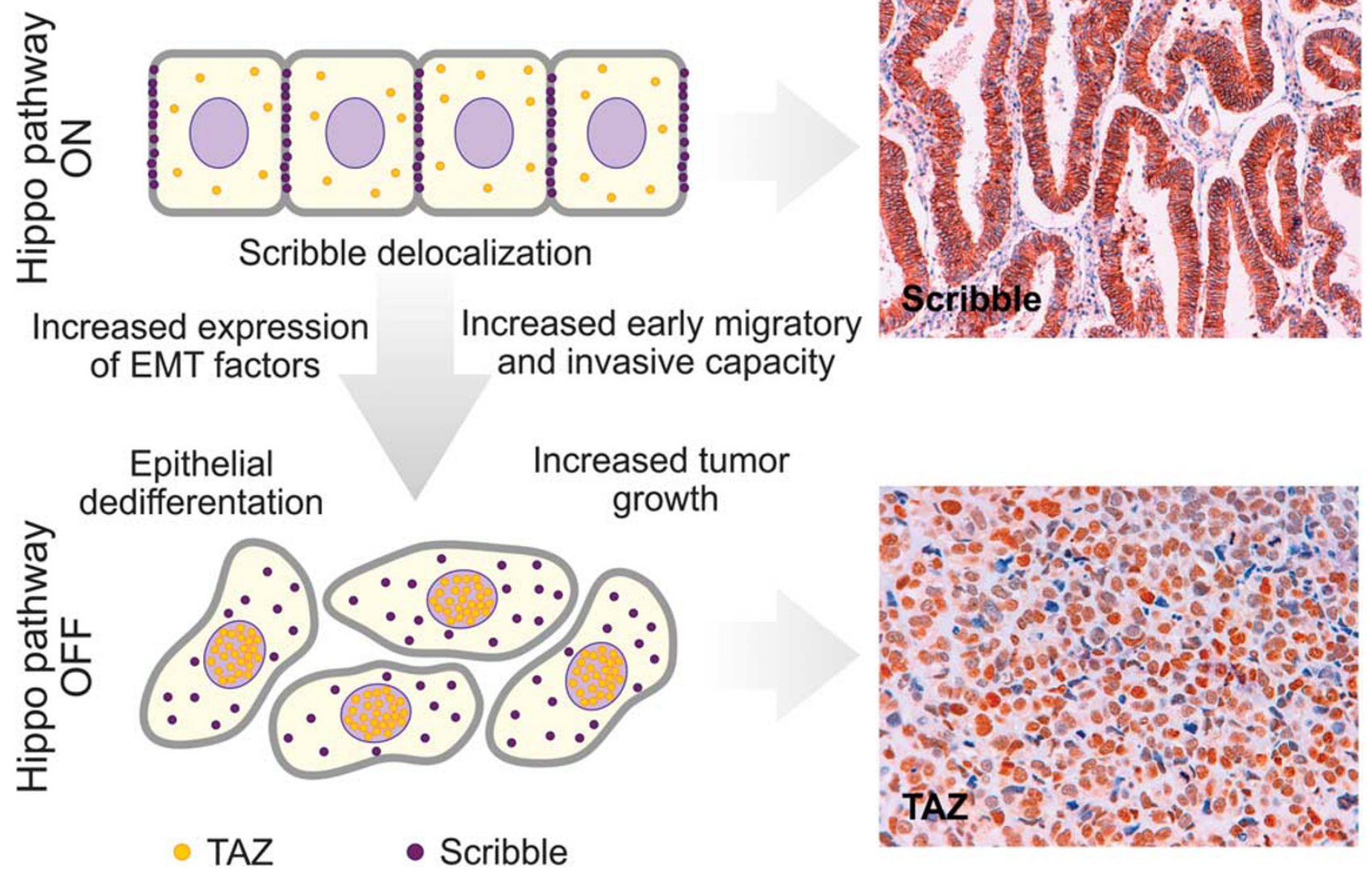

Figure 6 Scheme of the role of the Hippo pathway on the behavior of endometrial cancer cells (based on the mechanism proposed by Cordenonsi et al. ${ }^{18}$ Cell, 2011), showing Scribble expression on an endometrioid endometrial carcinoma and TAZ expression on an undifferentiated endometrial carcinoma.

TAZ overexpression is associated with a loss of E-cadherin while augmenting the $\mathrm{N}$-cadherin protein in the endometrial cancer cell lines, and that TAZ silencing decreases vimentin expression while increasing that of claudin-1. The loss of E-cadherin could be associated with the loss of Scribble activity, ${ }^{41}$ consistent with the effects on cell polarity and the triggering of epithelial to mesenchymal transition provoked by TAZ overexpression. In addition to this loss of epithelial features, we detected a negative correlation between the expression of miR-200s and WWTR1, showing that miR-200s expression is dampened during the transition from endometrioid endometrial carcinoma to endometrial carcinosarcoma, while WWTR1 expression increases. This relationship is reinforced by the significant correlation found between miR-200s expression and the expression of HMGA2, SNAI, SNAI2, TWIST1, ZEB1, and ZEB2 mRNA in the tumors analyzed, some of which are directly correlated with WWTR1 expression.

In summary, TAZ expression is associated with the most aggressive forms of endometrial carcinoma, such as undifferentiated endometrial carcinoma, endometrial carcinosarcoma, or endometrial serous carcinoma. In these tumors, TAZ expression is at least partially modulated by the expression and subcellular location of Scribble. TAZ overexpression favors the expression of epithelial to mesenchymal transition-related factors, and it induces an increase in the early migratory and invasive capacity of cells. Moreover, TAZ also favors tumor growth in vivo (Figure 6). These findings reveal a previously unknown role for the Hippo pathway in the development and progression of different subtypes of endometrial carcinoma.

\section{Acknowledgments}

We thank Ana Santos, Ma José Hernández and $M^{\mathrm{a}}$ Luisa Pecero of the Department of Pathology at the Instituto de Biomedicina de Sevilla-Hospital Universitario Virgen del Rocío (Sevilla), and Vanesa Santos and Saleta Morales from the Department of Biochemistry, Universidad Autónoma de MadridInstituto de Investigaciones Biomédicas 'Alberto Sols' (CSIC-UAM), IdiPAZ (Madrid) for their technical assistance. We would also like to thank Dr Wanjin Hong (Cancer and Developmental Cell Biology Division, Institute of Molecular and Cell Biology, Agency for Science, Technology and Research $A^{*}$ STAR, Singapur) for kindly providing 
us with the pBabe-puro-flag TAZ vector. This work was supported by grants from Spanish Ministry of Health, Instituto de Salud Carlos III (RD12/0036/ 0064 and PI13/02477 to JP and RETICC RD12/0036/ 0007 and PI13/00132 to GM-B); the European Development Regional Fund, 'A way to achieve Europe' EDRF to JP; the Community of Madrid (S2010/BMD-2303) and AECC Foundation to GMB; and by TV3 La Marató-2013 to GM-B and JP. LR-P is a recipient of a PFIS fellowship (Grant No. F109/00193). PG-S is funded by the AECC-2011. AM is a predoctoral student supported by a FPU fellowship (Spanish Ministry of Education, Culture and Sport). JD-M is a $\mathrm{PhD}$ researcher funded by the Consejería de Salud, Junta de Andalucía (PI0581/2009). MH-R is a $\mathrm{PhD}$ researcher funded by Community of Madrid. MAC is a $\mathrm{PhD}$ researcher funded by the ISCIII (RD06/0020/0013).

\section{Disclosure/conflict of interest}

The authors declare no conflict of interest.

\section{References}

1 Allard JE, Maxwell GL. Race disparities between black and white women in the incidence, treatment, and prognosis of endometrial cancer. Cancer Control 2009;16: 53-56.

2 López-García MA, Vieites MB, Castilla MA et al. Genetics of endometrial carcinoma. In: Ulrich P (ed). Cancer Genomics: Molecular Classification, Prognosis and Response Prediction, 1st edition. Springer Science and Business Media: Dordrecht. 2013;11:390.

3 Castilla MA, Moreno-Bueno G, Romero-Perez L et al. Micro-RNA signature of the epithelial-mesenchymal transition in endometrial carcinosarcoma. J Pathol 2011;223:72-80.

4 Romero-Perez L, Lopez-Garcia MA, Diaz-Martin J et al. ZEB1 overexpression associated with E-cadherin and microRNA-200 downregulation is characteristic of undifferentiated endometrial carcinoma. Mod Pathol 2013;26:1514-1524.

5 Mirantes C, Espinosa I, Ferrer I et al. Epithelial-tomesenchymal transition and stem cells in endometrial cancer. Hum Pathol 2013;44:1973-1981.

6 Colas E, Pedrola N, Devis L et al. The EMT signaling pathways in endometrial carcinoma. Clin Transl Oncol 2012;14:715-720.

7 Mani SA, Guo W, Liao MJ et al. The epithelialmesenchymal transition generates cells with properties of stem cells. Cell 2008;133:704-715.

8 Puisieux A, Brabletz T, Caramel J. Oncogenic roles of EMT-inducing transcription factors. Nat Cell Biol 2014;16:488-494.

9 De Craene B, Berx G. Regulatory networks defining EMT during cancer initiation and progression. Nat Rev Cancer 2013;13:97-110.

10 Morel AP, Lievre M, Thomas C et al. Generation of breast cancer stem cells through epithelialmesenchymal transition. PLoS One 2008;3:e2888.

11 Brabletz T, Jung A, Spaderna $\mathrm{S}$ et al. Opinion: migrating cancer stem cells-an integrated concept of malignant tumour progression. Nat Rev Cancer 2005;5: 744-749.

12 Moroishi T, Hansen CG, Guan KL. The emerging roles of YAP and TAZ in cancer. Nat Rev Cancer 2015;15: 73-79.

13 Hiemer SE, Varelas X. Stem cell regulation by the Hippo pathway. Biochim Biophys Acta 2013;1830:2323-2334.

14 Mo JS, Park HW, Guan KL. The Hippo signaling pathway in stem cell biology and cancer. EMBO Rep 2014;15:642-656.

15 Diepenbruck M, Waldmeier L, Ivanek R et al. Tead2 expression levels control the subcellular distribution of Yap and Taz, zyxin expression and epithelial-mesenchymal transition. J Cell Sci 2014;127:1523-1536.

16 Lei QY, Zhang H, Zhao B et al. TAZ promotes cell proliferation and epithelial-mesenchymal transition and is inhibited by the hippo pathway. Mol Cell Biol 2008;28:2426-2436.

17 Kanai F, Marignani PA, Sarbassova D et al. TAZ: a novel transcriptional co-activator regulated by interactions with 14-3-3 and PDZ domain proteins. EMBO J 2000;19:6778-6791.

18 Cordenonsi M, Zanconato F, Azzolin L et al. The Hippo transducer TAZ confers cancer stem cell-related traits on breast cancer cells. Cell 2011;147:759-772.

19 Piccolo S, Cordenonsi M, Dupont S. Molecular pathways: YAP and TAZ take center stage in organ growth and tumorigenesis. Clin Cancer Res 2013;19: 4925-4930.

20 Liu Y, Xin Y, Ye F et al. Taz-tead1 links cell-cell contact to zeb1 expression, proliferation, and dedifferentiation in retinal pigment epithelial cells. Invest Ophthalmol Vis Sci 2010;51:3372-3378.

21 Romero-Perez L, Castilla MA, Lopez-Garcia MA et al. Molecular events in endometrial carcinosarcomas and the role of high mobility group AT-hook 2 in endometrial carcinogenesis. Hum Pathol 2013;44:244-254.

22 Kononen J, Bubendorf L, Kallioniemi A et al. Tissue microarrays for high-throughput molecular profiling of tumor specimens. Nat Med 1998;4:844-847.

23 Zordan A. Fluorescence in situ hybridization on formalin-fixed, paraffin-embedded tissue sections. Methods Mol Biol 2011;730:189-202.

24 Livak KJ, Schmittgen TD. Analysis of relative gene expression data using real-time quantitative PCR and the 2(-Delta Delta C(T)) Method. Methods 2001;25: 402-408.

25 Moreno-Bueno G, Peinado H, Molina $\mathrm{P}$ et al. The morphological and molecular features of the epithelialto-mesenchymal transition. Nat Protoc 2009;4:1591-1613.

26 Franci C, Takkunen M, Dave N et al. Expression of Snail protein in tumor-stroma interface. Oncogene 2006;25:5134-5144.

27 Bhat KP, Salazar KL, Balasubramaniyan V et al. The transcriptional coactivator TAZ regulates mesenchymal differentiation in malignant glioma. Genes Dev 2011;25:2594-2609.

28 Yang N, Morrison CD, Liu P et al. TAZ induces growth factor-independent proliferation through activation of EGFR ligand amphiregulin. Cell Cycle 2012;11: 2922-2930.

29 Liu C, Huang W, Lei Q. Regulation and function of the TAZ transcription co-activator. Int J Biochem Mol Biol 2011;2:247-256.

30 de Cristofaro T, Di Palma T, Ferraro A et al. TAZ/ WWTR1 is overexpressed in papillary thyroid carcinoma. Eur J Cancer 2011;47:926-933. 
31 Koutsaki M, Spandidos DA, Zaravinos A. Epithelialmesenchymal transition-associated miRNAs in ovarian carcinoma, with highlight on the miR-200 family: prognostic value and prospective role in ovarian cancer therapeutics. Cancer Lett 2014;351:173-181.

32 Diaz-Lopez A, Moreno-Bueno G, Cano A. Role of microRNA in epithelial to mesenchymal transition and metastasis and clinical perspectives. Cancer Manag Res 2014;6:205-216.

33 Hua Y, Duan S, Murmann AE et al. miRConnect: identifying effector genes of miRNAs and miRNA families in cancer cells. PLoS One 2011;6:e26521.

34 Varelas X, Sakuma R, Samavarchi-Tehrani P et al. TAZ controls Smad nucleocytoplasmic shuttling and regulates human embryonic stem-cell self-renewal. Nat Cell Biol 2008;10:837-848.

35 Chan SW, Lim CJ, Guo K et al. A role for TAZ in migration, invasion, and tumorigenesis of breast cancer cells. Cancer Res 2008;68:2592-2598.
36 Dong J, Feldmann G, Huang J et al. Elucidation of a universal size-control mechanism in Drosophila and mammals. Cell 2007;130:1120-1133.

37 Zhang Y, Martens JW, Yu JX et al. Copy number alterations that predict metastatic capability of human breast cancer. Cancer Res 2009;69:3795-3801.

38 Kodaka M, Hata Y. The mammalian Hippo pathway: regulation and function of YAP1 and TAZ. Cell Mol Life Sci 2014;72:285-306.

39 Zhao B, Wei X, Li W et al. Inactivation of YAP oncoprotein by the Hippo pathway is involved in cell contact inhibition and tissue growth control. Genes Dev 2007;21:2747-2761.

40 Ouyang Z, Zhan W, Dan L. hScrib, a human homolog of Drosophila neoplastic tumor suppressor, is involved in the progress of endometrial cancer. Oncol Res 2010;18: 593-599.

41 Halaoui R, McCaffrey L. Rewiring cell polarity signaling in cancer. Oncogene 2014;34:939-950.

Supplementary Information accompanies the paper on Modern Pathology website (http://www.nature.com/ modpathol) 